\title{
Note
}

\section{Comparison of the Effects of Differently Processed Soy Foods on Lipid Metabolism in Rats}

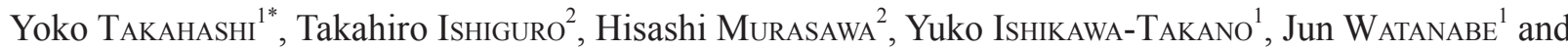 \\ Kohji YAMAKI $^{1}$ \\ ${ }^{I}$ Division of Food Function Research, Food Research Institute, NARO, 2-1-12, Kannondai, Tsukuba, Ibaraki 305-8642, \\ Japan \\ ${ }^{2}$ Food Research Laboratory, Asahimatsu Foods Co., Ltd., 1008 Dashina, Iida, Nagano 399-2561, Japan
}

Received June 17, 2016 ; Accepted October 18, 2016

\begin{abstract}
We investigated how different processing of soy foods impacts their hypolipidemic properties. Male rats were fed a diet containing $300 \mathrm{~g} / \mathrm{kg}$ of roasted soybean, powdered soy milk, dried fresh tofu, or kori tofu for 21 days. Roasted soybean, a fiber-rich food, significantly increased the fecal total lipid content. The fecal excretion of bile acids was significantly stimulated by fresh tofu and kori tofu, both of which contain coagulated protein. Soy milk and tofu products, which are relatively rich in protein, partly suppressed hepatic lipogenesis compared to roasted soybean. Unfortunately, the impact of these differences was not apparent in reducing lipid levels in serum and liver. Given these observations, constant consumption of each soy food may modulate intestinal lipid absorption and hepatic lipogenesis differently.
\end{abstract}

Keywords: food processing, lipid metabolism, nutritional composition, physicochemical property, soy foods

\section{Introduction}

Various hypolipidemic components of soybean and their functional mechanisms have been identified (Potter, 1995). As well as favorable components, soybean contains endogenous inhibitors of digestive enzymes and sources of undesirable flavors (Friedman and Brandon, 2001). Thus, adequate preparation of soybean products is necessary for human or animal ingestion. In Japan and some Asian countries, various cooking and processing methods have been developed for soy products. The wide variety of soy foods contributes to the promotion of soybean consumption. According to epidemiological studies, frequent consumption of soy products is associated with better lipid profiles in Asian populations (Lukito, 2001). Food processing also affects the nutritional composition of soy products (Lokuruka, 2011). Moreover, physicochemical modification of nutrients, such as heat denaturation and enzymatic hydrolysis. These modifications have been exploited to improve the functionality of foods, such as in the production of hypoallergenic foods (Sathe and Sharma, 2009). Taking into account the difference in metabolic state among individuals, food processing could be used to produce customized functional soy foods to alleviate dyslipidemia. A clear understanding of the differences in the functionality of each soy food could allow individuals to make their own appropriate choice to improve specific pathways of lipid metabolism through their daily diet. Furthermore, natural or common soy foods are more acceptable and accessible than newly developed functional foods. However, food is much more complex than a single component, yet it is not as well explored and more difficult to research (Jacobs and Tapsell, 2013). In an attempt to address this issue, we investigated food functionalities by directly comparing the nutritional characteristics of common soy foods in Japan and examined the influences of food processing on lipid metabolism in rats. 
Table 1. Composition of experimental diets

\begin{tabular}{|c|c|c|c|c|}
\hline & Roasted soybean & Soy milk & Fresh tofu & Kori tofu \\
\hline & \multicolumn{4}{|c|}{$(\mathrm{g} / \mathrm{kg}$ diet $)$} \\
\hline Dried soy foods & 300 & 300 & 300 & 300 \\
\hline Casein $^{a}$ & 88.8 & 62.4 & 40.1 & 38.2 \\
\hline Cornstarch & 150 & 150 & 150 & 150 \\
\hline Sucrose & 372.6 & 369.3 & 413.5 & 419.0 \\
\hline Corn oil $^{b}$ & 38.1 & 24.6 & 1.8 & 0.0 \\
\hline Cellulose & 0.0 & 43.2 & 44.1 & 42.3 \\
\hline Mineral mixture $^{c}$ & 35.0 & 35.0 & 35.0 & 35.0 \\
\hline Vitamin mixture $^{\mathrm{d}}$ & 10.0 & 10.0 & 10.0 & 10.0 \\
\hline L-Cystine ${ }^{b}$ & 3.0 & 3.0 & 3.0 & 3.0 \\
\hline \multirow[t]{2}{*}{ Chorine bitartrate ${ }^{\mathrm{b}}$} & 2.5 & 2.5 & 2.5 & 2.5 \\
\hline & \multicolumn{4}{|c|}{$(\mathrm{kJ} / \mathrm{kg}$ diet $)$} \\
\hline Energy & 16973 & 16359 & 16308 & 16210 \\
\hline
\end{tabular}

${ }^{a}$ The crude protein content was $88.1 \%$ (Wako Pure Chemical Industries, Osaka, Japan).

${ }^{\mathrm{b}}$ Wako Pure Chemical Industries (Osaka, Japan).

${ }^{c}$ AIN-93G mixture (Oriental Yeast, Tokyo, Japan).

${ }^{\mathrm{d}}$ AIN-93 mixture (Oriental Yeast, Tokyo, Japan).

\section{Materials and Methods}

Preparation of soy foods Roasted soybean, soy milk, soybean curd (fresh tofu), and freeze-dehydrated soybean curd (kori tofu) (Codex, 1995) were prepared by Asahimatsu Foods Co., Ltd. (Nagano, Japan). Whole soybeans were roasted at $130^{\circ} \mathrm{C}$ for $30 \mathrm{~min}$ in a steam convection oven (KEDC Multimax 6-23; Eloma, Maisach, Germany), crushed with a grinder (Grindomix; Retsch, Haan, Germany), and sifted through a 24-mesh screen. To prepare soy milk, soybeans were soaked in water at $15^{\circ} \mathrm{C}$ for 8 hours, then ground into a homogenate with fresh water using a blender (M-5A; NARA Machinery Co., Ltd., Tokyo, Japan) at $3300 \mathrm{rpm}$. The homogenate was heated to $>95^{\circ} \mathrm{C}$ for $3 \mathrm{~min}$ and filtered using a press equipped with a screen mesh (Twin Meister; Yanagiya Machinery Co., Ltd., Yamaguchi, Japan). A portion of the soy milk was rapidly freeze-dried and crushed as the sample. The other portion was mixed with calcium chloride solution to a concentration of $13 \mathrm{mM}$ calcium to form a curd. Half of the curd was rapidly freeze-dried, crushed, and sifted to provide the fresh tofu sample. The other half of the curd was slowly frozen at $-9^{\circ} \mathrm{C}$ for 3 hours and aged at $-2{ }^{\circ} \mathrm{C}$ for 20 days. The aged curd was thawed in water, dehydrated, dried in a hot-air dryer, and then crushed and sifted to give the kori tofu sample. The nutritional composition of the soy foods was analyzed by the Food Analysis Technology Center (Mie, Japan).

Animals and diets The animal study was approved by the Review Board of Animal Ethics of the Food Research Institute, NARO and performed in accordance with the institutional guidelines for the care and use of laboratory animals. Four-weekold male Sprague-Dawley rats were purchased from Charles River
(Kanagawa, Japan). Animals were housed individually in stainlesssteel cages in environmentally controlled conditions of temperature $\left(22-24^{\circ} \mathrm{C}\right)$, humidity $(55-65 \%)$, and 12 -hour light-dark cycle (07:00 - 19:00), and acclimatized to a commercial diet (Type NMF; Oriental Yeast, Tokyo, Japan) for 5 days. Animals were divided into four groups $(n=7-8)$ with similar average body weights, and assigned to one of the experimental diets. The dried soy foods at $300 \mathrm{~g} / \mathrm{kg}$ were added to the diets in the present experiment; this amount of soy foods was shown in our preliminary experiment using soy flour to modulate serum lipid levels (Takahashi, unpublished data). The experimental diets were prepared based on AIN-93G (Reeves et al., 1993) (Table 1). In this study, sucrose, used in AIN-76 (American Institute of Nutrition, 1977), was employed as a major carbohydrate source. Because it is known that sucrose can increase the serum triacylglycerol level and hepatic lipogenesis compared to starch in rats (Naismith and Rana, 1974), we expected that soy foods could effectively alleviate these changes. Casein, corn oil, and cellulose were added to the diets to adjust the protein, fat, and dietary fiber contents, respectively. Animals had free access to the diets and tap water for 21 days. To investigate how processing of soy, such as heat denaturation, affects lipid metabolism, a soy-free control diet was not included in this study.

Collection and preparation of samples Feces were collected for 3 days prior to the experimental endpoint. The lyophilized feces were weighed and stored at $-30^{\circ} \mathrm{C}$. At the end of the feeding period, rats were deprived of food for 3 hours and euthanized by collecting blood from the abdominal aorta during isoflurane anesthesia. Serum was separated by centrifugation and stored at 
Table 2. Nutritional composition and energy content in dried soy foods

\begin{tabular}{lcccc}
\hline & Roasted soybean & Soy milk & Fresh tofu & Kori tofu \\
\hline & \multicolumn{5}{c}{$(\mathrm{g} / 100 \mathrm{~g}$ dried food) } \\
Moisture & 6.0 & 4.8 & 5.4 & 8.0 \\
Protein & 34.9 & 43.2 & 50.2 & 50.7 \\
Fat & 22.0 & 26.5 & 34.1 & 34.7 \\
Carbohydrate & 32.4 & 20.3 & 6.5 & 3.0 \\
Ash & 4.7 & 5.2 & 3.8 & 3.6 \\
Total dietary fiber $^{\mathrm{a}}$ & 17.8 & 3.4 & 3.1 & 3.7 \\
(Water soluble) $_{\text {(Water insoluble) }}$ & $(1.9)$ & $(1.0)$ & $(0.8)$ & $(0.8)$ \\
& $(15.9)$ & $(2.4)$ & $(2.3)$ & $(2.9)$ \\
\hline \multirow{2}{*}{ Energy } & 1952 & $(\mathrm{~kJ} / 100 \mathrm{~g}$ dried food) & 2202 \\
\hline
\end{tabular}

${ }^{\text {a }}$ Total dietary fiber is the sum of water soluble and insoluble fibers.

$-30^{\circ} \mathrm{C}$. The liver was removed carefully and weighed. A portion of fresh liver was homogenized in buffer containing $0.25 \mathrm{M}$ sucrose, $3 \mathrm{mM}$ Tris- $\mathrm{HCl}$, and $1 \mathrm{mM}$ EDTA for the analysis of hepatic enzyme activities. For analysis of mRNA expression, another portion of the liver was homogenized with guanidine thiocyanate buffer (Chomczynski and Sacchi, 1987). The homogenates were stored at $-30^{\circ} \mathrm{C}$ until analysis. The remainder of the tissue was frozen in liquid nitrogen and stored at $-80^{\circ} \mathrm{C}$.

Analytical procedures for lipid metabolism Serum lipid concentrations were analyzed using commercial kits (Wako Pure Chemical Industries, Osaka, Japan). Liver lipids were extracted and analyzed using methods described previously (Takahashi and Ide, 2008). Total lipids in the feces were determined as described previously (Kaplan and Greenwood, 1998). Fecal bile acids were analyzed using hydroxysteroid dehydrogenase (Eaton and Klaassen, 1976). The activities of the enzymes involved in fatty acid metabolism were measured spectrophotometrically (Takahashi and Ide, 2008). Hepatic total RNA was extracted and complementary DNA was obtained (Takahashi and Ide, 2008). mRNA levels were analyzed using the real-time semi-quantitative reverse transcription polymerase chain reaction as described previously (Takahashi and Konishi, 2011). mRNA abundance was calculated as a ratio of the $\beta$-actin value in each cDNA sample and expressed as a percentage of the value obtained in rats fed the roasted soybean diet. The nucleotide sequences of the primers and probes have been reported previously (Lim et al., 2007; Takahashi, 2011).

Isoflavone content in soy foods and serum Isoflavone contents in the soy foods were determined according to the method reported previously (Ogita et al., 2015). Serum isoflavone concentrations were determined after treatment with $\beta$-glucuronidase/sulfatase in accordance with the method described previously (Takahashi and Ide, 2008). Isoflavones were calculated as aglycone levels.

Statistical analyses A power analysis was performed using $G^{*}$ Power 3.1.9.2 to determine the number of animals needed in order to detect significant differences among the groups (effect size
$=0.80 ; \alpha=0.05 ;$ power $=0.90)($ Faul et al., 2007). Statistical analyses were performed using SPSS $^{\circledR}$ Statistics 22 (IBM Japan, Tokyo, Japan). A one-way ANOVA followed by Tukey-Kramer's post hoc analysis was used to determine the significance of the dietary effects on the parameters examined. Values are presented as the mean $\pm \mathrm{SD}$. Significant differences were defined at a level of $p<0.05$.

\section{Results and Discussion}

Nutrient composition of soy foods Although the food processing did not considerably alter the nutritional compositions, protein and fat contents were higher in the following order on a dry weight basis: kori tofu $=$ fresh tofu $>$ soy milk $>$ roasted soybean (Table 2). Dietary fiber was more abundant in roasted soybean than in the other soy foods. These differences may be caused by the processing (Lokuruka, 2011), i.e., heating, filtration, extracting water-soluble components, and removal of whey.

Effect of soy foods on fecal lipid levels No significant differences were observed in body weight gain, food intake, or feeding efficacy (Table 3). Daily fecal weights were similar among the groups (Table 3). The fecal excretion of total lipids in the roasted soybean group was more than 2 -fold greater than that of the other groups. Total fiber contents in the diets were adjusted with cellulose (Table 1); however, the amounts of soluble and insoluble fiber in roasted soybean were approximately 2 -fold and more than 5-fold greater, respectively, than those in other soy foods (Table 2). Soybeans contain various fibers such as pectin, hemicellulose, and cellulose (Karr-Lilienthal et al., 2005). Choi et al. (1988) reported that soluble fiber of high molecular weight was more effective for the fecal excretion of lipids than insoluble cellulose. Thus, the ability of soy fiber to bind lipids may be greater than that of cellulose. This observation suggested that fiber-rich soy food is more potent for excreting excess lipids.

Kori tofu has a sponge-like texture that is generated by the slow-freezing and aging of soy protein at subzero temperatures (Ishiguro et al., 2011). One plausible cause is a resistant fraction of 
Table 3. The effect of the soy foods on growth and fecal lipid levels

\begin{tabular}{lcccc}
\hline & Roasted soybean & Soy milk & Fresh tofu & Kori tofu \\
\hline Growth & & & & \\
Body weight gain (g/day) & $9.63 \pm 1.46$ & $8.19 \pm 0.98$ & $8.11 \pm 0.67$ & $8.59 \pm 0.67$ \\
Food intake (g/day) & $20.3 \pm 1.4$ & $18.7 \pm 1.2$ & $18.7 \pm 1.1$ & $19.5 \pm 1.5$ \\
$\quad$ Feeding efficacy (g/g) & $2.14 \pm 0.22$ & $2.30 \pm 0.2$ & $2.31 \pm 0.1$ & $2.22 \pm 0.1$ \\
Feces & & & & \\
Dry fecal weight (g/day) & $1.58 \pm 0.18$ & $1.61 \pm 0.20$ & $1.45 \pm 0.08$ & $1.51 \pm 0.17$ \\
Fecal lipids (mg/day) & $251 \pm 34^{\mathrm{b}}$ & $100 \pm 15^{\mathrm{a}}$ & $100 \pm 8^{\mathrm{a}}$ & $111 \pm 13^{\mathrm{a}}$ \\
Fecal bile acids (mg/day) & $39.5 \pm 3.3^{\mathrm{a}}$ & $40.4 \pm 2.8^{\mathrm{a}}$ & $56.9 \pm 4.3^{\mathrm{b}}$ & $56.1 \pm 4.9^{\mathrm{b}}$ \\
\hline
\end{tabular}

${ }^{\mathrm{a}, \mathrm{b}}$ Values in a row with different superscript letters denote significant differences $(p<0.05)$.

Table 4. The effect of the soy foods on the activities and mRNA levels of lipogenic enzymes in livers

\begin{tabular}{lcccl}
\hline & Roasted soybean & Soy milk & Fresh tofu & Kori tofu \\
\hline Enzyme activities (nmol/min per mg protein) & & & & \\
$\quad$ Fatty acid synthase & $9.61 \pm 4.53^{\mathrm{b}}$ & $5.02 \pm 2.04^{\mathrm{ab}}$ & $7.21 \pm 3.27^{\mathrm{ab}}$ & $4.84 \pm 2.25^{\mathrm{a}}$ \\
$\quad$ ATP-citrate lyase & $29.2 \pm 7.8$ & $22.3 \pm 3.8$ & $21.9 \pm 4.1$ & $24.2 \pm 3.4$ \\
$\quad$ Glucose 6-phospate dehydrogenase & $19.2 \pm 4.9^{\mathrm{b}}$ & $15.3 \pm 2.5^{\mathrm{ab}}$ & $12.8 \pm 3.0^{\mathrm{a}}$ & $12.8 \pm 3.0^{\mathrm{a}}$ \\
$\quad$ & $29.8 \pm 9.9$ & $25.5 \pm 3.9$ & $24.9 \pm 5.2$ & $20.8 \pm 6.1$ \\
Malic enzyme & & & & \\
mRNA levels (relative expression level) & $100 \pm 68$ & $79.3 \pm 54.2$ & $63.9 \pm 28.2$ & $53.5 \pm 24.4$ \\
$\quad$ Fatty acid synthase & $100 \pm 26$ & $93.0 \pm 35.8$ & $83.1 \pm 22.4$ & $77.4 \pm 16.4$ \\
ATP-citrate lyase & $100 \pm 13^{\mathrm{b}}$ & $80.8 \pm 7.1^{\mathrm{a}}$ & $73.6 \pm 9.7^{\mathrm{a}}$ & $69.0 \pm 7.3^{\mathrm{a}}$ \\
Glucose 6-phospate dehydrogenase & $100 \pm 31^{\mathrm{b}}$ & $80.4 \pm 8.8^{\mathrm{ab}}$ & $69.1 \pm 13.7^{\mathrm{a}}$ & $65.3 \pm 15.4^{\mathrm{a}}$ \\
$\quad$ Malic enzyme & $100 \pm 17$ & $96.5 \pm 16.4$ & $103 \pm 19$ & $84.1 \pm 18.5$ \\
SREBP-1c &
\end{tabular}

ATP, adenosine triphosphate; SREBP-1, sterol regulatory element-binding protein-1

${ }^{\mathrm{a}, \mathrm{b}}$ Values in a row with different superscript letters denote significant differences $(p<0.05)$.

soy protein hydrolysate, the high-molecular-weight fraction (HMF) (Sugano et al., 1990). These manufacturing processes may affect the protein structure of kori tofu, thereby increasing the content of HMF that would bind a greater amount of bile acid than soy protein isolate (Ishiguro et al., 2011). Therefore, we anticipated that the HMF would account for the differences in fecal bile acid excretion between kori tofu and fresh tofu. However, both tofu diets increased the bile acid level similarly compared to the roasted soybean and soy milk diets (Table 3). It was suggested that protein coagulation, rather than the slow freezing and aging of the protein, may increase the content of HMF and facilitate the fecal excretion of bile acids. Alternatively, the freeze-drying process may make the coagulated protein less hydrolyzable. We also confirmed the expression of hepatic mRNAs involved in steroid metabolism: lowdensity lipoprotein receptor, 3-hydroxy-3-methylglutaryl (HMG)CoA reductase, cytochrome P450 family 7 subfamily A member 1 (Cyp7a1), and sterol regulatory element-binding protein (SREBP)-2. However, no significant differences were observed among the groups (data not shown). These results suggested that the increase in fecal bile acid excretion was not derived from the stimulation of internal cholesterol metabolism.

Effect of soy foods on hepatic fatty acid metabolism The activities and mRNA levels of hepatic enzymes related to fatty acid synthesis tended to be lower in the soy milk, fresh tofu, and kori tofu groups than in the roasted soybean group (Table 4). The differences were partly significant. Dietary soy protein was shown previously to reduce triglyceride levels in plasma and liver, and these effects were associated with marked reductions in the activities of hepatic lipogenic enzymes (Iritani et al., 1996). In this study, the protein content of soy foods (Table 1) was inversely correlated with the enzyme activities. Pearson's correlation coefficients were as follows: fatty acid synthase, $r=-0.386, p=$ 0.038; ATP citrate lyase, $r=-0.407, p=0.029$; glucose 6-phosphate dehydrogenase, $r=-0.628, p<0.001$; and malic enzyme, $r=$ $-0.421, p=0.023$. However, the enzyme activities did not necessarily correspond to the mRNA levels of the enzymes and the transcription factor SREBP-1c. In view of the fact that the differences in these parameters were only partly significant, the mechanism for the better hypolipidemic activities of protein-rich soy foods remains unclear at present. The enzyme activities involved in fatty acid $\beta$-oxidation were not affected by the soy foods (data not shown).

Effect of soy foods on serum and hepatic lipid levels Triacylglycerol and cholesterol concentrations in the serum tended 
Table 5. The effect of the soy foods on serum and liver lipids

\begin{tabular}{|c|c|c|c|c|}
\hline & Roasted soybean & Soy milk & Fresh tofu & Kori tofu \\
\hline \multicolumn{5}{|c|}{ Serum lipid concentrations $(\mathrm{mmol} / \mathrm{L})$} \\
\hline Triacylglycerol & $2.35 \pm 0.68$ & $1.86 \pm 0.97$ & $1.67 \pm 0.40$ & $2.25 \pm 0.58$ \\
\hline Cholesterol & $2.11 \pm 0.60$ & $1.82 \pm 0.29$ & $1.58 \pm 0.18$ & $1.89 \pm 0.32$ \\
\hline \multicolumn{5}{|c|}{ Liver lipid concentrations $(\mu \mathrm{mol} / \mathrm{g})$} \\
\hline Triacylglycerol & $24.4 \pm 9.3$ & $22.1 \pm 8.6$ & $17.3 \pm 4.1$ & $18.5 \pm 5.7$ \\
\hline Cholesterol & $1.98 \pm 0.42$ & $2.10 \pm 0.38$ & $1.97 \pm 0.27$ & $2.05 \pm 0.51$ \\
\hline
\end{tabular}

A

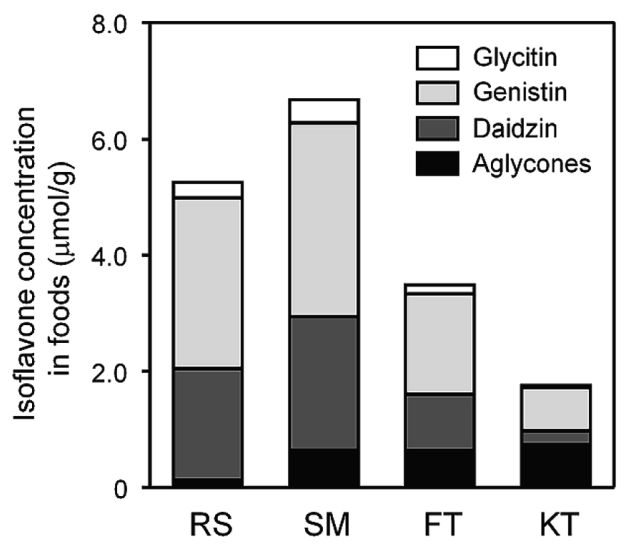

B

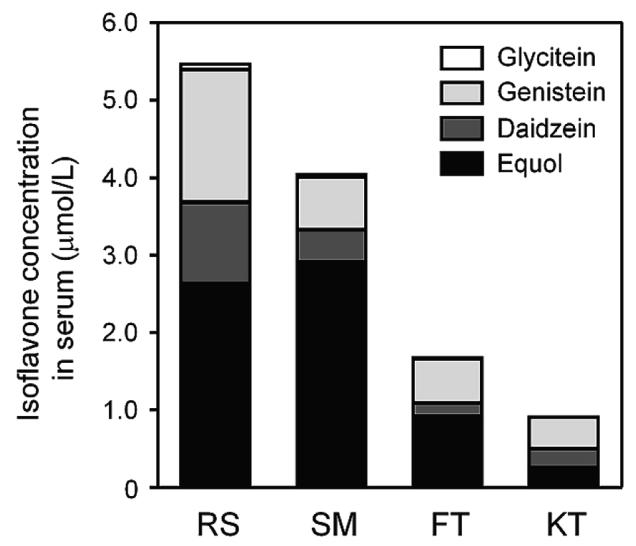

Fig. 1. Isoflavone levels in soy foods (A) and serum of rats fed the experimental diets containing soy foods (B). RS, roasted soybean; SM, soy milk; FT, fresh tofu; KT, kori tofu. A, Data were determined in a duplicate analysis and expressed as the mean value of aglycone equivalents. B, Values were expressed as the mean of 7-8 animals per group.

to be lower in the soy milk and tofu groups than in the roasted soybean group; however, the differences were not significant (Table 5). With regard to the relationship with protein content in the soy foods, only hepatic triacylglycerol levels were inversely correlated $(r=-0.337, p=0.044)$. The present study suggested that the hypolipidemic properties of these soy foods were generally similar, although serum lipid levels were more clearly decreased in rats fed kori tofu than in those fed a non-soy diet (Takahashi and Konishi, 2011). The differences in the amounts of fecal lipid and bile acid excretions may be negligible in modulating serum and hepatic lipid levels. In fact, a large proportion of dietary fat was absorbed, according to the calculation of the daily excretions; $88.1 \%$ for the roasted soybean group and $94.5-94.9 \%$ for the other groups.

Isoflavone levels in soy products and serum of rats fed soy foods We also examined the probability of isoflavones in soy foods regulating lipid metabolism. Total isoflavones were most abundant in soy milk, followed by roasted soybean, fresh tofu, and kori tofu (Fig. 1A). Meanwhile, total isoflavone levels in serum were highest in rats fed roasted soybean (Fig. 1B). Among the isoflavones, daidzein derivatives and their metabolite equol accounted for a significant proportion. Equol was relatively abundant in the soy milk group compared to the roasted soybean group. In the fresh tofu group, most of the daidzein derivatives were also converted into equol. Although the soy foods altered isoflavone levels in the bloodstream, they did not affect lipid metabolism in the present study.

\section{Conclusion}

Food processing influenced the protein, fat, and fiber composition as well as the protein conformation of soy foods. The impact of these differences was not apparent on lipid levels in serum and liver; however, fecal lipid levels and hepatic lipogenesis were partly but significantly affected by the type of soy foods. Given these observations, constant consumption of each soy food may modulate intestinal lipid absorption and hepatic lipogenesis differently.

Acknowledgments The authors thank Michiko Abe, Yoshiko Toyama, and Michiko Yamamoto for technical assistance. This study was supported by Kieikai Research Foundation, Japan, and a Research Project on Development of Agricultural Products and Foods with Health-Promoting Benefits (NARO), Japan.

\section{References}

American Institute of Nutrition. (1977). Report of the American Institute of 
Nutrition ad hoc committee on standards for nutritional studies. J. Nutr., 107, 1340-1348.

Choi, Y.S., Cho, S.H., Kim, H.J., and Lee, H.J. (1998). Effects of soluble dietary fibers on lipid metabolism and activities of intestinal disaccharidases in rats. J. Nutr. Sci. Vitaminol., 44, 591-600.

Chomczynski, P. and Sacchi, N. (1987). Single-step method of RNA isolation by acid guanidinium thiocyanate-phenol- chloroform extraction. Anal. Biochem., 162, 156-159.

Codex. (1995). Codex general standard for food additives. In "CODEX STAN 192-1995" ed. by Food and Agriculture Organization of United Nations, Annex B, pp 31.

Eaton, D.L. and Klaassen, C.D. (1976). Effects of acute administration of taurocholic and taurochenodeoxycholic acid on biliary lipid excretion in the rat. Proc. Soc. Exp. Biol. Med., 151, 198-202.

Faul, F., Erdfelder, E., Lang, A.G., and Buchner, A. (2007). G*Power 3: A flexible statistical power analysis program for the social, behavioral, and biomedical sciences. Behav. Res. Methods, 39, 175-191.

Friedman, M. and Brandon, D.L. (2001). Nutritional and health benefits of soy proteins. J. Agric. Food Chem., 49, 1069-1086.

Iritani, N., Hosomi, H., Fukuda, H., Tada, K., and Ikeda, H. (1996). Soybean protein suppresses hepatic lipogenic enzyme gene expression in Wistar fatty rats. J. Nutr., 126, 380-388.

Ishiguro, T., Tatsunokuchi, S., Mitusi, N., Kayahara, H., Murasawa, H., Konishi, Y., and Nagaoka, S. (2011). Cholesterol-lowering effect of koritofu protein and its high-molecular-weight fraction content. Biosci. Biotechnol. Biochem., 75, 575-577.

Jacobs, D.R. and Tapsell, L.C. (2013). Food synergy: the key to a healthy diet. Proc. Nutr. Soc., 72, 200-206.

Kaplan, R.J. and Greenwood, C.E. (1998). Poor digestibility of fully hydrogenated soybean oil in rats: a potential benefit of hydrogenated fats and oils. J. Nutr., 128, 875-880.

Karr-Lilienthal, L.K., Kadzere, C.T., Grieshop, C.M., and Fahey, G.C. Jr. (2005). Chemical and nutritional properties of soybean carbohydrates as related to nonruminants: a review. Livest. Prod. Sci., 97, 1-12.

Lim, J.S., Adachi, Y., Takahashi, Y., and Ide, T. (2007). Comparative analysis of sesame lignans (sesamin and sesamolin) in affecting hepatic fatty acid metabolism in rats. Br. J. Nutr., 97, 85-95.
Lokuruka, M.N.I. (2011). Effects of processing on soybean nutrients and potential impact on consumer health: an overview. Afr. J. Food Agric. Nutr. Dev., 11, 5000-5017.

Lukito, W. (2001). Candidate foods in the Asia-Pacific region for cardiovascular protection: nuts, soy, lentils and tempe. Asia Pac. J. Clin. Nutr., 10, 128-133.

Naismith, D.J. and Rana, I.A. (1974). Sucrose and hyperlipidaemia. I. The relationship between the plasma lipid concentrations and enzymes of tissue lipogenesis. Ann. Nutr. Metab., 16, 238-248.

Ogita, T., Watanabe, J., Wakagi, M., Nakamichi, K., Komiyma, S., Takebayashi, J., Mano, J., Kitta, K., Koyano, S., and Takano-Ishikawa, Y. (2015). Evaluation of a method to quantify isoflavones in soybean by single and multi-laboratory validation studies. Food Sci. Tech. Res., 21, 473-477.

Potter, S.M. (1995). Overview of proposed mechanisms for the hypocholesterolemic effect of soy. J. Nutr., 125, 606S-611S.

Reeves, P.G., Nielsen, F.H., and Fahey, G.C. Jr. (1993). AIN-93 purified diets for laboratory rodents: final report of the American Institute of Nutrition ad hoc writing committee on the reformulation of the AIN-76A rodent diet. J. Nutr., 123, 1939-1951.

Sathe, S.K. and Sharma, G.M. (2009). Effects of food processing on food allergens. Mol. Nutr. Food Res., 53, 970-978.

Sugano, M., Goto, S., Yamada, Y., Yoshida, K., Hashimoto, Y., Matsuo, T., and Kimoto, M. (1990). Cholesterol-lowering activity of various undigested fractions of soybean protein in rats. J. Nutr., 120, 977-985.

Takahashi, Y. and Ide, T. (2008). Effects of soy protein and isoflavone on hepatic fatty acid synthesis and oxidation and mRNA expression of uncoupling proteins and peroxisome proliferator-activated receptor gamma in adipose tissues of rats. J. Nutr. Biochem., 19, 682-693.

Takahashi, Y. (2011). Soy protein and fish oil independently decrease serum lipid concentrations but interactively reduce hepatic enzymatic activity and gene expression involved in fatty acid synthesis in rats. $J$. Nutr. Sci. Vitaminol., 57, 56-64.

Takahashi, Y. and Konishi, T. (2011). Tofu (soybean curd) lowers serum lipid levels and modulates hepatic gene expression involved in lipogenesis primarily through its protein, not isoflavone, component in rats. J. Agric. Food Chem., 59, 8976-8984. 